\title{
Obtención de Biodiesel por Transesterificación Alcalina a partor de Aceites Vegetales Residual en Lima
}

\author{
Biodiesel by Transesterification Alkaline from Waste Vegetable \\ Oils in Lima
}

\author{
Linda N. Zavaleta Palomino ${ }^{1 *}$, Jean Pierre A. Suavo Carrión ${ }^{2}$ \\ ${ }^{1}$ Facultad de Ingeniería Geográfica, Ambiental y Ecoturismo, Universidad Nacional Federico Villarreal, Lima, Perú
}

\begin{abstract}
RESUMEN
El objetivo de esta investigación es conocer el proceso de producción óptimo para generar biodiesel, por transesterificación alcalina, a partir de aceites vegetales residuales de los restaurantes del distrito de San Borja, Lima- Perú. Para ello, se analizó el aceite vegetal residual recolectado, se determinó la concentración de metanol (\%v/v), la concentración de hidróxido de potasio (\%p/p), el tiempo de reacción óptimo, y por último se determinó la calidad del biodiesel producido.

Los resultados mostraron que es posible realizar biodiesel con el aceite recolectado, ya que su grado de acidez $(1,56 \%)$ fue inferior al 3\%. Las condiciones óptimas para lograr la máxima conversión de la reacción se obtuvieron cuando se usó una concentración de metanol del 30\%, una concentración de hidróxido de potasio del $0,4 \%$ respecto al peso del aceite y un tiempo de reacción de $3 \mathrm{hrs} 30 \mathrm{~min}$ a una temperatura constante de $60^{\circ} \mathrm{C}$. Bajo estas condiciones se obtuvo un rendimiento de biodiesel del 85,97\%. Al biodiesel obtenido bajo las mejores condiciones de reacción se le analizaron cuatro propiedades del combustible, encontrándose que la viscosidad cinemática fue 5,5 cSt, el número de acidez fue 0,68 mgKOH/g, la ceniza sulfatada fue 0,0478 \% y el carbón conradson fue $0,142 \%$.
\end{abstract}

Palabras claves.- Transesterificación alcalina, grado de acidez, máxima conversión número de acidez, viscosidad cinemática, ceniza sulfatada, carbón conradson y postratamiento del biodiesel.

\begin{abstract}
In this paper, it is presented an experiment carried out with the objective of knowing the optimum production process in order to generate bio diesel by alkaline transesterification, from residual vegetable oils from the Restaurants in San Borja. In order to do so, first of all of the residual vegetal oil collected was analyzed, then it was determined the concentration of methanol $(\% \mathrm{v} / \mathrm{v})$, the concentration of potassium hydroxide $(\% \mathrm{p} / \mathrm{p})$ and the optimum reaction time, and lastly, it was determined the bio diesel quality produced.

The results showed that it is possible to generate biodiesel from the collected oil, due to its grade of acidity $(1,56 \%)$ was lower than $3 \%$. The optimum conditions to get the maximum conversion of the reaction were achieved when it was used a methanol concentration of $30 \%$, a concentration of potassium hydroxide of $0,4 \%$ regarding the weight of the oil and a reaction time of 3:30 minutes at a constant temperature of $60 \%$. Under these conditions it was obtained a performance of biodiesel of $85,97 \%$. Biodiesel obtained under the best reaction conditions will be analyzed four fuel properties, finding that the kinematic viscosity was 5,5 cSt, the acid number was $0,68 \mathrm{mg} \mathrm{KOH} \mathrm{/} \mathrm{g,} \mathrm{the} \mathrm{sulfated} \mathrm{ash} \mathrm{was} \mathrm{0,0478 \%} \mathrm{and} \mathrm{Conradson} \mathrm{Carbon} \mathrm{was} \mathrm{0,142 \% .}$
\end{abstract}

Key Words.- Alkaline Transesterification, grade of acidity, maximum conversion, number of acidity, kinematic viscosity, sulfated ash, conradson carbon and after treatment of biodiesel.

\section{INTRODUCCIÓN}

La bioenergía se define como la energía que sale de la biomasa, es decir, es energía producida a partir de materiales orgánicos.

Estos materiales orgánicos pueden tener su origen en cultivos energéticos, residuos forestales orgánicos municipales, excrementos de animales, grasas y aceites de origen vegetal o animal.

De los triglicéridos contenidos en las grasas animales y vegetales se puede derivar un combustible de origen orgánico llamado biodiesel.

El biodiesel es un biocombustible producido a partir de la reacción de transesterificación entre un aceite vegetal, ya sea este virgen o usado, un alcohol y un catalizador.

El biodiesel obtenido a partir de aceites vegetales residuales o usados puede reemplazar parcialmente al diesel derivado del petróleo. Al ser elaborado con aceites usados se da una alternativa al reciclaje de este material que por lo general es desechado a las 


\begin{tabular}{|c|c|c|c|}
\hline \multicolumn{4}{|c|}{ Cuadro 1. Condiciones de reacción en la transesterificación ${ }^{1}$} \\
\hline Parámetros & & Valor & \\
\hline Concentración del metanol respecto al aceite (\%v/v) & $30 \%$ & $40 \%$ & $50 \%$ \\
\hline Concentración del KOH respecto al aceite (\%p/p) & $0.4 \%$ & $0.65 \%$ & $1.0 \%$ \\
\hline Temperatura $\left({ }^{\circ} \mathrm{C}\right)$ & & 60 & \\
\hline Tiempo de reacción (min) & & 120 & \\
\hline
\end{tabular}

alcantarillas o como basura común, generando un gran problema de contaminación ambiental.

El presente trabajo está orientado específicamente al estudio del proceso de producción óptimo para generar biodiesel, por transesterificación alcalina, a partir de aceites vegetales residuales de los restaurantes del distrito de San Borja, Lima-Perú. Para ello se analizó el aceite recolectado, se determinó la concentración óptima del metanol $(\% \mathrm{v} / \mathrm{v})$, del hidróxido de potasio $(\% \mathrm{p} / \mathrm{p})$ y del tiempo de reacción, por último se determinó la calidad del biodiesel obtenido comparándolo con las especificaciones técnicas nacionales e internacionales.

Esta investigación fue importante llevarla a cabo porque permitió conocer el proceso óptimo en la producción del biodiesel, para que haya una máxima conversión de los triglicéridos en metilésteres o formación de biodiesel.

\section{PARTE EXPERIMENTAL}

La recolección de aceite se realizó durante el mes de Mayo del 2013.

El aceite recolectado se mezcló en un cilindro para obtener una muestra homogénea.

El aceite recolectado fue pre-tratado con el fin de obtener un aceite libre de impurezas y de agua. Este pretratamiento consistió en tres operaciones: decantación, filtrado y eliminación de agua.

Al aceite recolectado, libre de impurezas y agua, se le estableció una unidad experimental de 200 $\mathrm{ml}$, en la cual se le midió una serie de propiedades fisicoquímicas: ${ }^{\mathrm{a}}$ Densidad $(\mathrm{g} / \mathrm{ml})$, Viscosidad cinemática (cSt), Potencial de hidrógeno, Índice de Acidez (mg KOH/g muestra) y Índice de Peróxido $\left(\mathrm{meqO}_{2} / \mathrm{kg}\right)$.

\section{NIVEL LABORATORIO}

Una vez caracterizado el aceite, se procedió a la realización de pruebas a nivel de laboratorio. Estas pruebas se realizaron a diferentes condiciones de reacción que aparecen en la Cuadro 1, con la finalidad de determinar a qué concentración de catalizador (KOH) y alcohol (metanol) se obtiene mejores resultados de producción, para posteriormente elaborarlo a nivel piloto en la planta Kallpa Wasi de la Municipalidad de San Borja, Lima-Perú.

La calidad del producto obtenido en las pruebas a nivel de laboratorio, se evaluó mediante la determinación de los siguientes parámetros ${ }^{\mathrm{b}}$ : Rendimiento del producto final (biodiesel), Rendimiento del glicerol, Densidad, Viscosidad cinemática e Índice de acidez.

La calidad del biodiesel se calificará al margen de la normativa nacional e internacional.

Una vez que se ha verificado que los parámetros de viscosidad, densidad e índice de acidez se encuentran entre los rangos aceptables por la normativa de calidad de biodiesel, se procede a realizar un análisis de varianza (ANOVA), con una prueba estadística Tukey para cada parámetro más el rendimiento de biodiesel y glicerina, con la finalidad de comparar y determinar si tienenuna diferencia significativa o son homogéneos las reacciones.

\section{NIVEL PILOTO}

La transesterificación a nivel piloto, se realizó en el reactor de la planta Kallpa Wasi para ello se utilizó la concentración del metanol y la concentración del catalizador óptimos de la reacción en las pruebas a nivel delaboratorio.

A las muestras o ensayos se le midieron los parámetros que ayudarían a identificar el tiempo óptimo de reacción ${ }^{\mathrm{a}}$ : Rendimiento del producto final (biodiesel), Rendimiento del glicerol, Densidad, Viscosidad cinemática e Índice de acidez.

\footnotetext{
La Revista Científica TECNIA protege los derechos de autor bajo la Licencia 4.0 de Creative Commons: Attribution 4.0 International (CC BY 4.0).
}

a Estos parámetros fueron hallados en el laboratorio de química orgánica de la Facultad de Ciencias Naturales y ftatemáticas de la Universidad Nacional Federico Villarreal

b Estos niveles se han definido teniendo en cuenta los resultados de diferentes investigaciones.

c Parámetros hallados utilizando la metodología de la tesis doctoral de Azócar, 2010.

d Estos parámetros fueron hallados por el Laboratorio de Energía Renovables de la Universidad Nacional Agraria La ftolina. 
Al producto final (biodiesel), se le midieron las propiedades que caracterizan a un combustible, entre ellas ${ }^{\mathrm{d}}$ : Cenizas sulfatadas, Carbón conradson, Viscosidad cinemática e Índice de acidez.

\section{RESULTADOS Y DISCUSIONES}

El total recolectado de aceites de 29 restaurantes fue de 172,15 L de los cuales solo se utilizó 50,6 kg para la transesterificación a nivel de laboratorio y transesterificación a nivel piloto.

El aceite recolectado, por ser procedente de restaurantes, contenía impurezas y agua como se aprecia en el Tabla 1. Del aceite inicial, el 3,4 kg fue de sólidos gruesos, el 0,071 kg fue de sólidos finos y el $0,054 \mathrm{~kg}$ fue de agua.

Siendo así, solo el 47,075 kg de aceite fue apto para producir biodiesel. Esta cantidad fue utilizada para la transesterificación a nivel laboratorio y piloto.

Tabla 1. Cantidades de cada operación del pretratamiento

\begin{tabular}{lc}
\hline Operaciones & Cantidades (kg) \\
\hline Aceite Inicial & 50,6 \\
Decantación (sólidos gruesos) & 3,4 \\
Filtrado (sólidos finos) & 0,071 \\
Aceite libre de impurezas & 47,129 \\
Agua & 0,054 \\
Aceite libre de agua & 47,075 \\
\hline
\end{tabular}

Al aceite libre de impurezas y agua, se le analizó algunos parámetros como la viscosidad cinemática, densidad, pH, índice de acidez, índice de peróxido y humedad. De los cuales los más representativos son el índice de acidez, que indica el contenido de ácidos grasos libres (se recomienda que para el proceso de transesterificación el aceite tenga AGL inferiores al
Tabla 2. Parámetros medidos en el aceite.

\begin{tabular}{|c|c|c|c|}
\hline PARÁMETROS & UNIDADES & ACEITE & $\begin{array}{c}\text { NTP } \\
\text { 209.001:1983 } \\
\text { (revisada el } \\
\text { 2012) } \\
\end{array}$ \\
\hline Viscosidad a $40^{\circ} \mathrm{C}$ & cSt o $\mathrm{mm}^{2} / \mathrm{s}$ & 5,68 & - \\
\hline Densidad a $15^{\circ} \mathrm{C}$ & $\mathrm{g} / \mathrm{ml}$ & 0,90 & - \\
\hline $\mathrm{pH}$ & Adimensional & 4,59 & - \\
\hline Grado de Acidez & $\%$ & 1,56 & - \\
\hline Índice de Acidez & mg KOH/g aceite & 0,3 & $<0.2$ \\
\hline $\begin{array}{l}\text { Índice de Pe- } \\
\text { róxido }\end{array}$ & meq $0^{2} / \mathrm{kg}$ & 4 & $<5$ \\
\hline Humedad & $\%$ & $\underline{0,115}$ & $\leq 0.1$ \\
\hline
\end{tabular}

$3 \%$ ), el índice de peróxido, que indica el grado de oxidación, y la humedad.

La NTP 209.001.1983, muestra valores que deben cumplir todos los aceites vegetales comestibles. En la Tabla 2 se observa que nuestro aceite recolectado tiene un exceso de AGL y un exceso de humedad con respecto a la norma peruana, indicando que el aceite debe ser desechado y no utilizado para consumo humano.

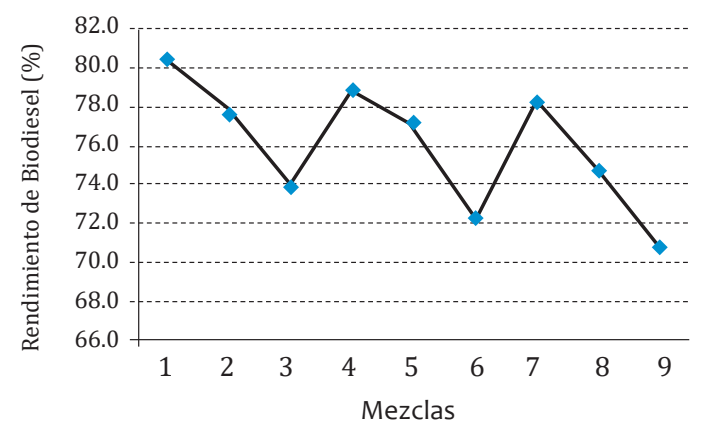

Figura 1. Distribución del rendimiento de biodiesel con respecto a cada mezcla

Tabla 3. Comparación del rendimiento de biodiesel a diferentes mezclas

\begin{tabular}{|c|c|c|c|c|c|c|}
\hline \multirow[t]{2}{*}{ Mezclas } & \multirow[t]{2}{*}{ № } & \multicolumn{5}{|c|}{ Subconjunto para alfa $=0.05$} \\
\hline & & 1 & 2 & 3 & 4 & 5 \\
\hline 9 & 3 & 70,8533 & & & & \\
\hline 6 & 3 & 72,2767 & 72,2767 & & & \\
\hline 3 & 3 & & 73,8400 & & & \\
\hline 8 & 3 & & 74,8333 & 74,8333 & & \\
\hline 5 & 3 & & & 77,2267 & 77,2267 & \\
\hline 2 & 3 & & & & 77,7067 & \\
\hline 7 & 3 & & & & 78,3667 & 78,3667 \\
\hline 4 & 3 & & & & 78,9100 & 78,9100 \\
\hline 1 & 3 & & & & & 80,5233 \\
\hline Sig. & & 0,615 & 0,057 & 0,087 & 0,411 & 0,154 \\
\hline
\end{tabular}




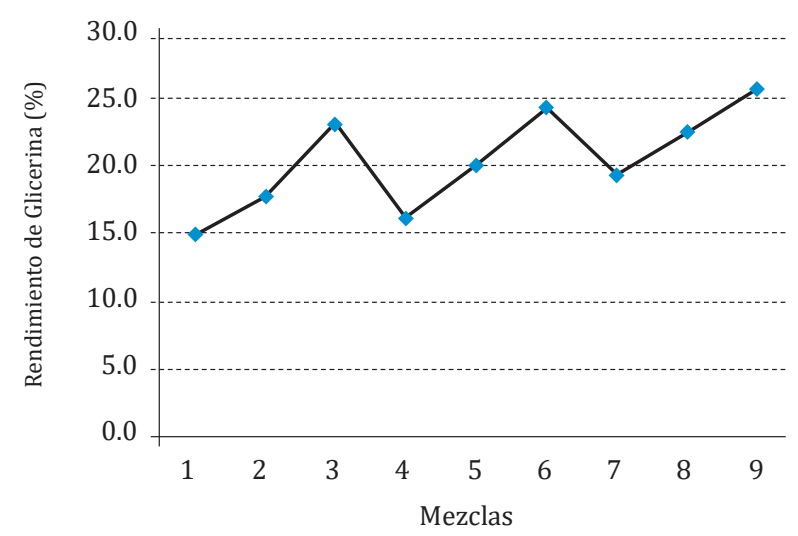

Figura 2. Distribución del rendimiento de glicerina con respecto a cada mezcla

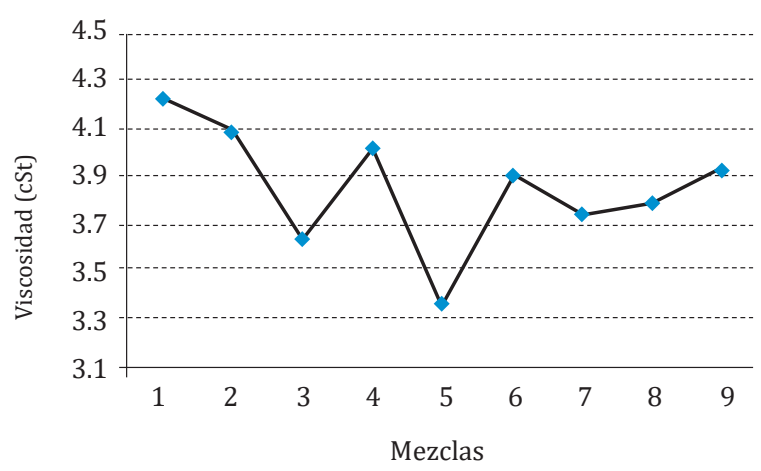

Figura 3. Distribución de la viscosidad cinemática con respecto a cada mezcla.

Tabla 4. Comparación del rendimiento de biodiesel a diferentes mezclas

HSO de Tukey

\begin{tabular}{|c|c|c|c|c|c|c|c|}
\hline \multirow{2}{*}{ Mezclas } & \multirow{2}{*}{ № } & \multicolumn{6}{|c|}{ Subconjunto para alfa $=0.05$} \\
\hline & & 1 & 2 & 3 & 4 & 5 & 6 \\
\hline 1 & 3 & 14,9393 & & & & & \\
\hline 4 & 3 & 15,9920 & & & & & \\
\hline 2 & 3 & & 17,6170 & & & & \\
\hline 7 & 3 & & 19,1240 & 19,1240 & & & \\
\hline 5 & 3 & & & 20,0187 & & & \\
\hline 8 & 3 & & & & 22,4333 & & \\
\hline 3 & 3 & & & & 22,9397 & 22,9397 & \\
\hline 6 & 3 & & & & & 24,2440 & 24,2440 \\
\hline 9 & 3 & & & & & & 25,5117 \\
\hline Sig & & 0,368 & 0,067 & 0,567 & 0,962 & 0,152 & 0,176 \\
\hline
\end{tabular}

Tabla 5. Comparación de la viscosidad cinemática a diferentes mezclas

HSD de Tukey

\begin{tabular}{ccc}
\hline Mezclas & № & $\begin{array}{c}\text { Subconjunto para alfa }=0.05 \\
1\end{array}$ \\
\hline 5 & 3 & 3,3600 \\
3 & 3 & 3,6267 \\
7 & 3 & 3,7400 \\
8 & 3 & 3,7967 \\
6 & 3 & 3,9133 \\
9 & 3 & 3,9267 \\
4 & 3 & 4,0167 \\
2 & 3 & 4,0967 \\
1 & 3 & 4,2233 \\
Sig & & 0,113 \\
\hline
\end{tabular}

Tabla 6. Comparación de la densidad a diferentes mezclas

HSD de Tukey

\begin{tabular}{ccc}
\hline Mezclas & № & $\begin{array}{c}\text { Subconjunto para alfa }=0.05 \\
1\end{array}$ \\
\hline 7 & 3 & 0,88500 \\
6 & 3 & 0,88567 \\
1 & 3 & 0,88667 \\
5 & 3 & 0,88667 \\
2 & 3 & 0,88733 \\
8 & 3 & 0,88833 \\
4 & 3 & 0,88900 \\
9 & 3 & 0,88900 \\
3 & 3 & 0,88900 \\
Sig & & 0,960 \\
\hline
\end{tabular}


Tabla 7. Comparaciones del índice de acidez a diferentes mezclas

\begin{tabular}{ccccc}
\hline \multirow{2}{*}{ Mezclas } & № & \multicolumn{3}{c}{ Subconjunto para alfa $=0.05$} \\
& & 1 & 2 & 3 \\
\hline 1 & 3 & 0,2300 & & \\
3 & 3 & 0,2433 & & \\
8 & 3 & 0,2433 & & \\
2 & 3 & 0,2500 & & \\
9 & 3 & 0,2767 & 0,2767 & \\
5 & 3 & 0,2933 & 0,2933 & \\
4 & 3 & 0,3067 & 0,3067 & \\
7 & 3 & & 0,3800 & 0,3800 \\
6 & 3 & & & 0,4467 \\
\hline Sig. & & 0,345 & 0,086 & 0,515
\end{tabular}

El índice de peróxido no sobrepasó los $5 \mathrm{meq} \mathrm{O}^{2} /$ $\mathrm{kg}$, por lo tanto el aceite aún no ha sido oxidado significativamente durante el proceso defritura.

Por otra parte el Grado de Acidez, que fue de 1,56\%, nos indica que el aceite sí es apto para la reacción de transesterificación, ya que menor al 3\% puede ser transesterificado por una sola etapa (alcalina)[1].

\section{Nivel laboratorio}

A nivel de laboratorio, se determinó la concentración óptima de metanol e hidróxido de potasio.

Una vez elaborado el biodiesel, para cada ensayo (Ver Cuadro 1), se procedió a realizar pruebas físicas tales como viscosidad, densidad, índice de acidez, rendimiento del producto final y rendimiento de glicerina, con el fin de determinar sus cualidades como combustible frente a estándares nacionales e internacionales.
Las Figuras 1, 2, 3, 4, 5 muestran las distribuciones gráficas de las medias y en las Tablas 3, 4, 5, 6, 7 las comparaciones de los rendimientos de biodiesel a través de la prueba de Tukey.

\section{NIVEL PILOTO}

En la producción de biodiesel a nivel piloto se utilizó $37 \mathrm{~kg}(41,1 \mathrm{~L})$ de aceite con la concentración de metanol $(\% \mathrm{v} / \mathrm{v})$ de $30 \%$, equivalente a $12,3 \mathrm{~L}$, y la concentración de $\mathrm{KOH}(\% \mathrm{p} / \mathrm{p})$ de $0,4 \%$, equivalente a $148 \mathrm{~g}$ que fueron los que produjeron mejores resultados de optimización a nivel de laboratorio, para luego determinar el tiempo óptimo de la reacción de transesterificación. Este valor del catalizador esta dentro del rango que establecen para catálisis básica [2], que es del $0,2 \%$ al 1,6\% en peso del aceite.

Para determinar la concentración de $\mathrm{KOH}$ y $\mathrm{CH}_{4} \mathrm{O}$ óptimos, no solo se basó en el rendimiento de

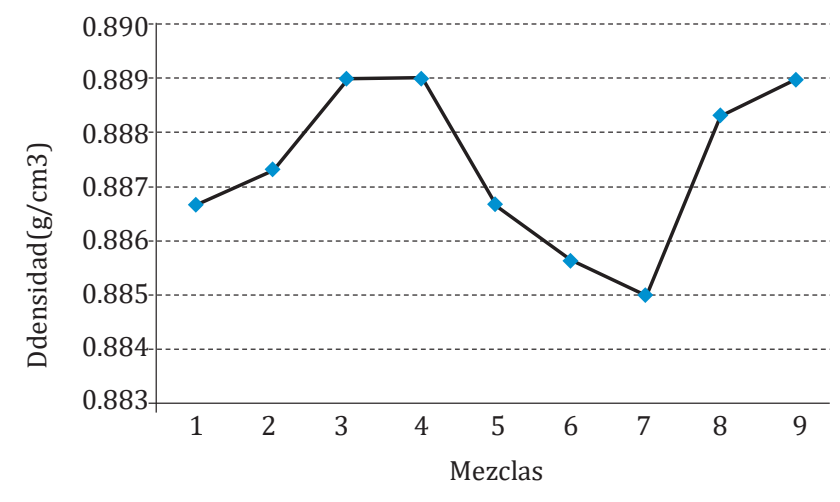

Figura 4. Distribución de la densidad con respecto a cada mezcla. 


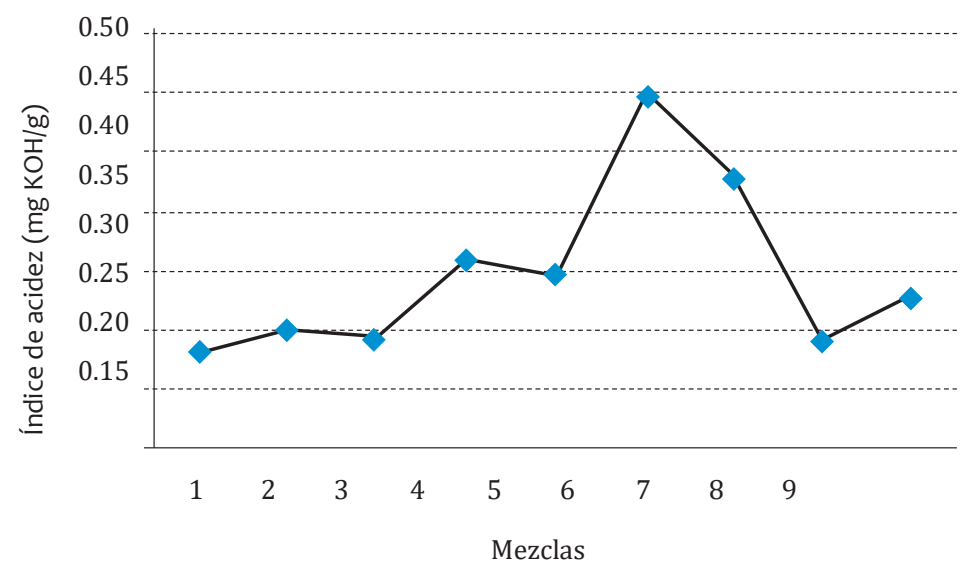

Figura 5. Distribución del índice de acidez con respecto a cada mezcla.

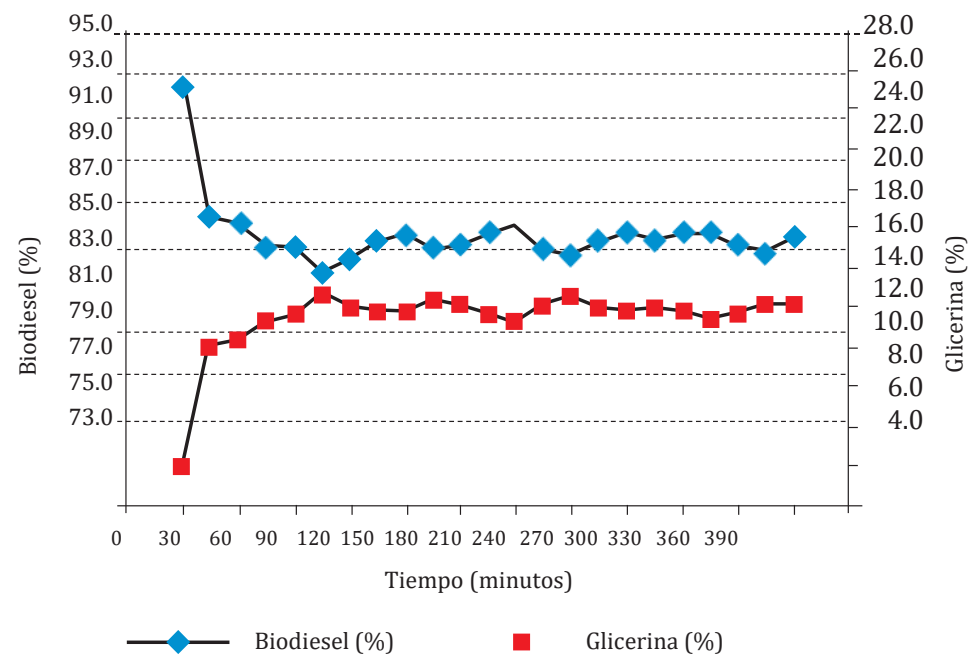

Figura 6. Rendimiento de biodiesel y glicerina

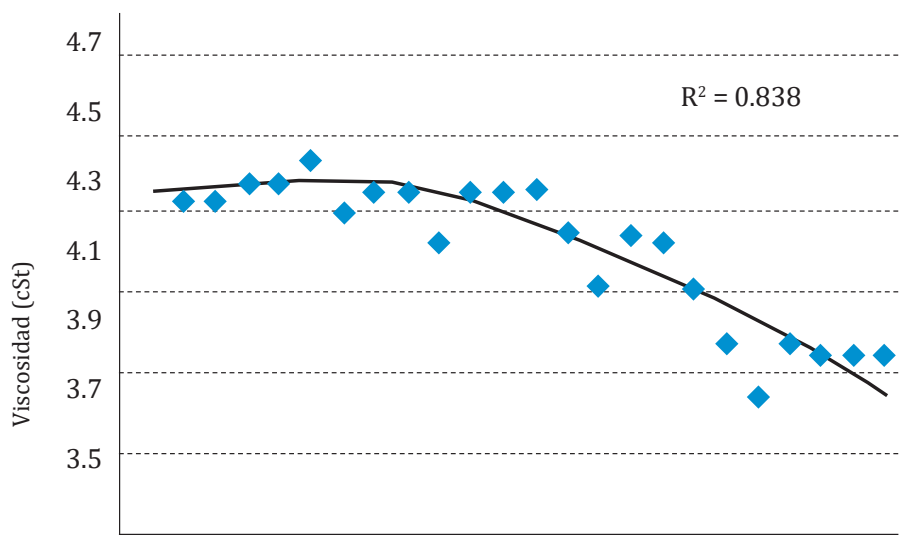

$0 \quad 30 \quad 60 \quad 90 \quad 120150180210240270300330360$

Tiempo (minutos)

Figura 7. Variación de la viscosidad cinemática con respecto a los tiempos 


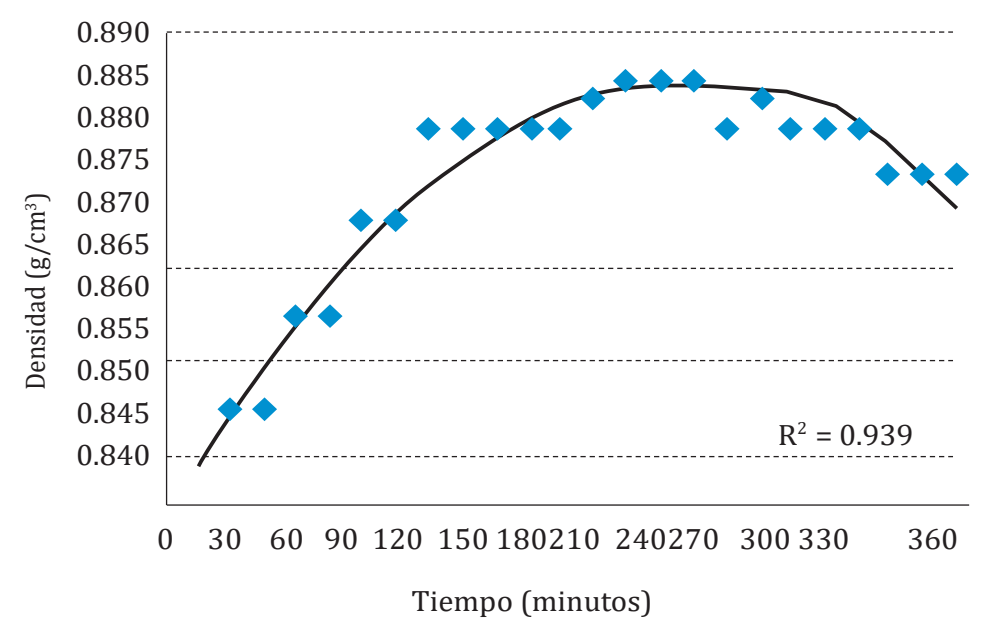

Figura 8. Variación de la densidad con respecto a los tiempos

biodiesel y glicerina sino también se utilizó la metodología analítica de la tesis doctoral de Azócar (2010). Se debe resaltar que para una mayor exactitud en el rendimiento se debió analizar el contenido de glicerina total, mono-, di-, trigliceridos y metilésteres, por cromatografía de gases a cada ensayo, como lo recomienda Nazario [3]. Pero por motivo de presupuesto no se pudo realizar el análisis.

Sin embargo, Nazario [3], Lafont[4] y Tirado [5] utilizaron solo viscosidad, densidad e índice de acidez, parámetros físicos para determinar el rendimiento de su producto final, dando a conocer que estos tres tres parámetros son representativos en la calidad del

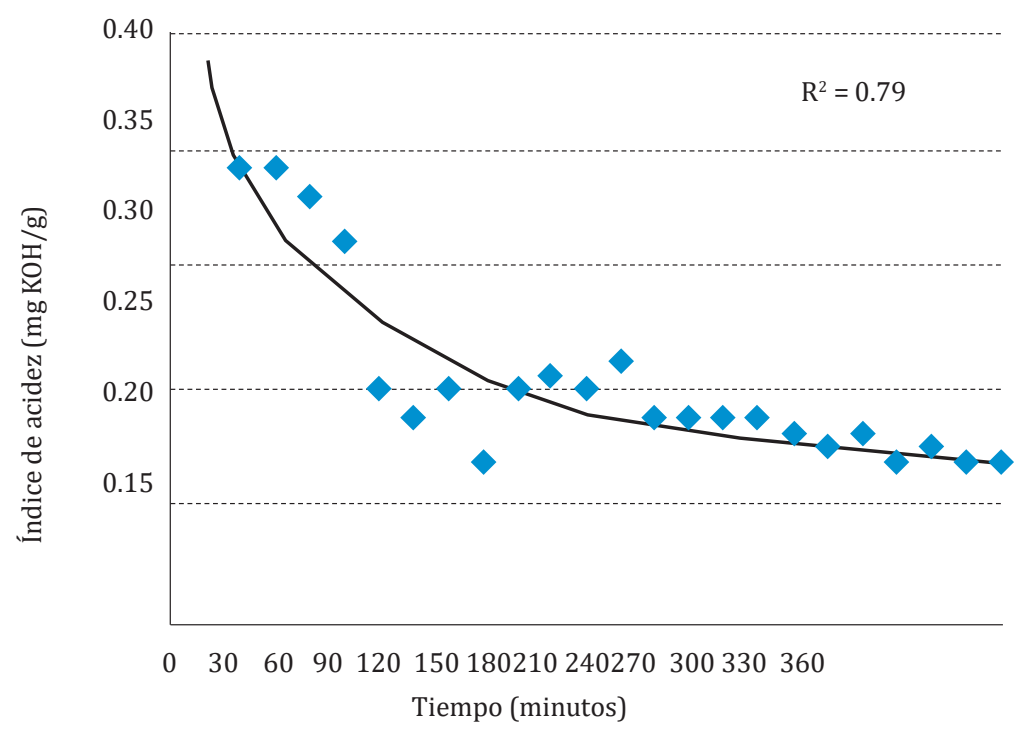

Figura 9. Variación de índice de acidez con respecto a los tiempos

biodiesel.

Para determinar el tiempo óptimo se halló los siguientretes parámetros respecto al tiempo: Rendimiento de Biodisel, viscosidad cinética, densidad y índice de acidez; como se muestran en las Figuras 6,7,8 y 9.
Al biodiesel obtenid o a las mejores condiciones, punto óptimo para la transesterificación (KOH: 0,4\%, CH4O: 30\%, Tiempo: 3 hrs 30min), se le realizó un análisis de su calidad en el laboratorio de Energía Renovables de la Universidad Nacional Agraria la Molina. Leung [6], dice que la conversión de triglicéridos en ésteres metílicos aumentan con el tiempo de reacción, 
Tabla 8. Propiedades del biodisel obtenido a las mejores condiciones

\begin{tabular}{|c|c|c|c|c|c|}
\hline \multirow[b]{2}{*}{ PROPIEDAD } & \multirow[b]{2}{*}{ VALOR } & \multicolumn{3}{|c|}{ ESPECIFICACIÓN TÉCNICA DEL BIODIESEL } & \multirow{2}{*}{$\begin{array}{l}\text { ESPECIF. DIESEL } \\
\text { NTP5 } \\
\text { 321.003:2005 } \\
\text { (Diesel 2 S-50) }\end{array}$} \\
\hline & & $\begin{array}{c}\text { NTP }^{2} \\
321.125: 2008\end{array}$ & $\begin{array}{c}\text { ASTM }^{3} \\
\text { D } 6751-7\end{array}$ & $\begin{array}{c}\mathrm{EN}^{4} \\
14214: 2003\end{array}$ & \\
\hline $\begin{array}{l}\text { Viscosidad Cinemática a } 40^{\circ} \mathrm{C} \\
\text { (cSt) }\end{array}$ & 5,5 & $1,90-6,0$ & $1,90-6,0$ & 5 máx. & $2,0-4,5$ \\
\hline Numero de Acidez (mg KOH/g) & 0,68 & 0,5 máx. & 0,5 máx. & 0,5 máx. & 0,08 máx. \\
\hline Cenizas sulfatadas (\%) & 0,0478 & 0,020 máx. & 0,020 máx. & 0,020 máx. & 0,01 máx. \\
\hline Carbón Conradson (\%) & 0,142 & 0,05 máx. & 0,05 máx. & 0,30 máx. & 0,3 máx. \\
\hline
\end{tabular}

sin embrago un tiempo excesivo no aumenta los rendimientos.

En la Tabla 10 se presentan estos valores, comparándolos con las normas internacionales y nacionales de calidad para el biodiesel y diesel.

\section{CONCLUSIONES}

$\mathrm{Al}$ analizar el aceite vegetal residual recolectado de los restaurantes del distrito de San Borja, se pudo conocer que no son aptos para consumo humano, ya que el índice de acidez y humedad $(0.3 \mathrm{mg} \mathrm{KOH} / \mathrm{g}$ y $0.115 \%$, respectivamente) exceden al valor de la NTP de Aceites Vegetales Comestibles $1<0.2 \mathrm{mg} \mathrm{KOH} / \mathrm{g}$ $\mathrm{y}<0.1 \%$, respectivamente), pero sí es apto para la elaboración del biodiesel, debido a que el grado de acidez (1.56\%) es menor al 3\%.

Con base en un diseño factorial de experimentos de $3 \times 3$, se pudo determinar que la máxima conversión de la reacción de transesterificación se alcanzó cuando se usó una concentración de metanol (\%v/v) de $30 \%$, una concentración de $\mathrm{KOH}(\% \mathrm{p} / \mathrm{p}$ ) de $0,4 \%$ y un tiempo de reacción de $3 \mathrm{hrs} .30 \mathrm{~min}$. Bajo estas condiciones se obtuvo un rendimiento de biodiesel de $85,97 \%$.

Las propiedades determinadas del biodiesel obtenido, han sido comparadas con las especificaciones técnicas nacionales e internacionales (NTP, ASTM y EN), concluyendo que la viscosidad cinemática $(5,5$ cSt) está dentro del margen de la NTP (1,90-6,0 cSt) y ASTM (1,90-6,0 cSt), a excepción de la norma EN (5 máx. cSt); el número de acidez $(0,68 \mathrm{mgKOH} / \mathrm{g})$ está fuera del rango de las normas $(0,5 \mathrm{mgKOH} / \mathrm{g}$ máx. $)$; la ceniza sulfatada $(0,0478 \%)$ tampoco cumple con lo establecido en las normas $(0,020 \%$ máx. $)$ y el carbón conradson $(0,142 \%)$ está dentro del valor de la norma EN (0,3 \% máx.), más no de la ASTM (0,05 \% máx.) y NTP $(0,05 \%$ máx.).
Al Dr. Walter Gómez Lora por la orientación, paciencia y tiempo a lo largo de la realización de esta investigación, a Jean Pierre Suavo Carrión por el cariño y motivación que me otorgó cada día, a Harry Rivera, Miguel Baltazar, Ing. Sonia Espinoza y Raúl Ortiz por darme su apoyo y conocimientos para el desarrollo de este trabajo.

A la Municipalidad de San Borja por permitirme realizar la tesis en su planta piloto de energía renovable.

\section{BIBLIOGRAFÍA}

[1] Yin J, Xiao M, Wang A and Xiu Z 2008 Síntesis de biodiesel a partir de aceite de soja por catálisis subcrítico con metanol

[2] Rojas A, Girón E and Torres H 2009 Variables de operación en el proceso de transesterificación de aceites vegetales: una revisión-catálisis química

[3] Ramírez N 2006 Aprovechamiento de aceites vegetales de desechos generados por el comedor universitario de la Universidad Nacional Agraria la Molina, para la elaboración de biodiesel Tesis de grado

[4] Lafont J, Páez M and Torres Y 2010 Análisis Químico de Mezclas Biodiesel de Aceite de Cocina Usado y Diesel por Espectroscopia Infrarroja

[5] Tirado J, Trejo F and Sotelo R 2010 Conversión del aceite no comestible a biodiesel

[6] Leung D and Guo Y 2006 La transesterificación del aceite de freír limpio y usados: Optimización de la producción de biodiesel 